\title{
LTH 383
}

\section{PROGRESS WITH LARGE $N_{f} \beta$-FUNCTIONS *}

\author{
J.A. GRACEY \\ Department of Mathematical Sciences, University of Liverpool, P.O. Box 147, \\ Liverpool, L69 3BX, United Kingdom
}

\begin{abstract}
We report on the progress in the computation of the $\beta$-functions of $\phi^{4}$ theory and QCD in the large $N$ expansion. For the former we give an analytic formula for the critical exponent which encodes higher order coefficients in the series beyond those currently known in the $\overline{\mathrm{MS}}$ scheme at $O\left(1 / N^{2}\right)$ in a large $N$ expansion. This allows us to deduce new coefficients in the field anomalous dimension which relate to Kreimer's knot theory of the divergences of a quantum field theory. A similar exponent is computed for the $\beta$-function of QCD at $O\left(1 / N_{f}\right)$.
\end{abstract}

\section{Introduction}

We report on two areas where progress has been made into revealing the higher order structure of $\beta$-functions using the large $N$ expansion. First, we recall that a highlight of the Pisa workshop was the breakthrough by Broadhurst in computing those diagrams of the six and seven loop $\beta$-function in $\phi^{4}$ theory which are free of subdivergences 1 This was in response to Kreimer's association of the divergences of multiloop Feynman diagrams involving transcendental numbers with knot theory and in particular positive braids $\mathrm{O}$ For example, the appearance of the Riemann zeta series, $\zeta(2 n-3) n \geq 3$, is well known in high order $\overline{\mathrm{MS}}$ calculations and these were associated with $(2 n-3,2)$ torus knots The six and seven loop terms of the $\beta$-function contained new transcendentals These were represented by double or triple sums which could not be reduced to products of $\zeta(n)$. They were associated with the torus knot $10_{124}$ and the hyperbolic knots $10_{139}, 10_{152}$ and $11_{353}$. As these interesting knot numbers appear at very high orders it is important to have an efficient way of accessing them. The large $N$ approach is a technique which facilitates this 3 For instance in $\phi^{4}$ theory with an $O(N)$ symmetry the field anomalous dimension, $\gamma(g)$, has been computed analytically in $d$-dimensions at $O\left(1 / N^{3}\right)$ at a fixed point, $g_{c}$, using a conformal bootstrap method $t$ The result involves a particular two loop Feynman integral whose $\epsilon$-expansion, where $d=4-2 \epsilon$, contains irreducible

${ }^{*}$ Talk presented at 5th International Workshop on Software Engineering, Neural Nets, Genetic Algorithms, Expert Systems, Symbolic Algebra and Automatic Calculations, Lausanne, Switzerland, 2-6th September, 1996. 
double sums. These therefore can be associated with the more complicated positive braids. To find at what order in perturbation theory they arise and to determine the explicit $\overline{\mathrm{MS}}$ coefficients in $\gamma(g)$ associated with them one needs to solve the critical renormalization group relation $\eta=2 \gamma\left(g_{c}\right)$. Necessary for this is the location of the critical coupling $g_{c}=g_{c}(\epsilon, N)$ which is dimensionless in 4-dimensions. In this article we report the result of the $O\left(1 / N^{2}\right)$ calculation of $\beta^{\prime}\left(g_{c}\right)$ and therefore $g_{c}$ which allows us to proceed. The remainder of the paper is devoted to a similar calculation of the $\beta$-function of QCD 5 though at $O\left(1 / N_{f}\right)$, which will provide new $\overline{\mathrm{MS}}$ coefficients and therefore an independent check for future 4 and higher loop calculations, albeit at the easier end of the range of perturbative calculability.

\section{$2 \quad \phi^{4} \beta$-function}

The large $N$ method to compute all orders coefficients of the renormalization group functions at a fixed point of the $\beta$-function has been discussed previously 6 G For $\phi^{4}$ theory with an $O(N)$ symmetry we use the lagrangian in the form

$$
L=\frac{1}{2}\left(\partial \phi^{i}\right)^{2}+\frac{1}{2} \sigma \phi^{i} \phi^{i}-\sigma^{2} / 2 g
$$

where $\sigma$ is an auxiliary field. This form is used so that uniqueness can be applied andalso to emphasis the equivalence with the $O(N) \sigma$ model in $(2-2 \epsilon)$ dimensions 1 This $d$-dimensional equivalence means that the critical exponents computed in either model are the same. Although various $\sigma$ model exponents aside from $\eta$ are known at $O\left(1 / N^{2}\right)$ the exponent $\omega$ which we define as $\omega=-$ $\beta^{\prime}\left(g_{c}\right) / 2$ has not been determined. It plays a role in understanding corrections to scaling and as it is not related through scaling laws it must be computed independently.

In the large $N$ formalisn this is achieved by considering corrections to asymptotic scaling and then solving the $\phi$ and $\sigma$ Schwinger Dyson equations self consistently. At leading order due to the structure of the $N$-dependence of the $2 \times 2$ matrix yielding $\omega$ there is one 2 loop and one 3 loop Feynman diagram to compute This situation is parallel to the same computation in the Gross Neveu model For the $O\left(1 / N^{2}\right)$ calculation, in addition to computing the $O(1 / N)$ corrections to the previous graphs, there are two 2-loop, eight 3-loop, eleven 4-loop, ten 5-loop and two 6-loop integrals which need to be evaluated. We find 3

$$
\omega_{1}=(2 \mu-1)^{2} \eta_{1}
$$


and

$$
\begin{aligned}
\omega_{2}=\eta_{1}^{2} & {\left[-\frac{4\left(\mu^{2}-5 \mu+5\right)(2 \mu-3)^{2}(\mu-1) \mu^{2}\left(\bar{\Phi}+\bar{\Psi}^{2}\right)}{(\mu-2)^{3}(\mu-3)}\right.} \\
- & \frac{16 \mu(2 \mu-3)^{2}}{(\mu-2)^{3}(\mu-3)^{2} \eta_{1}} \\
- & \frac{3\left(4 \mu^{5}-48 \mu^{4}+241 \mu^{3}-549 \mu^{2}+566 \mu-216\right)(\mu-1) \mu^{2} \hat{\Theta}}{2(\mu-2)^{3}(\mu-3)} \\
- & {\left[16 \mu^{10}-240 \mu^{9}+1608 \mu^{8}-6316 \mu^{7}+15861 \mu^{6}\right.} \\
& -25804 \mu^{5}+26111 \mu^{4}-14508 \mu^{3}+2756 \mu^{2} \\
& +672 \mu-144)] /\left[(\mu-2)^{4}(\mu-3)^{2}\right] \bar{\Psi} \\
+ & {\left[144 \mu^{14}-2816 \mu^{13}+24792 \mu^{12}-130032 \mu^{11}+452961 \mu^{10}\right.} \\
& \quad-1105060 \mu^{9}+1936168 \mu^{8}-2447910 \mu^{7}+2194071 \mu^{6} \\
& -1320318 \mu^{5}+460364 \mu^{4}-43444 \mu^{3}-26280 \mu^{2} \\
& \left.+8208 \mu-864] /\left[2(2 \mu-3)(\mu-1)(\mu-2)^{5}(\mu-3)^{2} \mu\right]\right]
\end{aligned}
$$

where $\eta_{1}=-4 \Gamma(2 \mu-2) /[\Gamma(2-\mu) \Gamma(\mu-1) \Gamma(\mu-2) \Gamma(\mu+1)], \bar{\Psi}(\mu)=\psi(2 \mu-3)$ $+\psi(3-\mu)-\psi(\mu-1)-\psi(1), \hat{\Theta}(\mu)=\psi^{\prime}(\mu-1)-\psi^{\prime}(1), \bar{\Phi}(\mu)=\psi^{\prime}(2 \mu-3)$ $-\psi^{\prime}(3-\mu)-\psi^{\prime}(\mu-1)+\psi^{\prime}(1)$ and $\omega=\sum_{i=0}^{\infty} \omega_{i} / N^{i}$ with $\omega_{0}=\mu-2$. The $\epsilon$-expansion of Eq. (3) near $d=4-2 \epsilon$ dimensipns gives total agreement with the coefficients of the explicit 5 -loop $\overline{\mathrm{MS}}$ results 10 So writing the $\beta$-function in the form where only the $O(1 / N)$ and $O\left(1 / N^{2}\right)$ coefficients are included,

$$
\beta(g)=\frac{1}{2}(d-4) g+\left(a_{1} N+b_{1}\right) g^{2}+\sum_{r=2}^{\infty}\left(a_{r} N+b_{r}\right) N^{r-2} g^{r+1}
$$

we find the new $\overline{\mathrm{MS}}$ coefficients

$$
\begin{aligned}
a_{6}= & {[29+528 \zeta(3)-432 \zeta(4)] / 1866240 } \\
a_{7}= & {[61+80 \zeta(3)+1584 \zeta(4)-1728 \zeta(5)] / 26873856 } \\
b_{6}= & {[-28160 \zeta(7)+95200 \zeta(6)-150336 \zeta(5)-6912 \zeta(4) \zeta(3)} \\
& \left.+14112 \zeta(4)+24064 \zeta^{2}(3)+11880 \zeta(3)+5661\right] / 466560 \\
b_{7}= & -7795200 \zeta(8)+32724480 \zeta(7)-43286400 \zeta(6)-3993600 \zeta(5) \zeta(3) \\
& +31998720 \zeta(5)-622080 \zeta^{2}(4)+8663040 \zeta(4) \zeta(3)+2538432 \zeta(4) \\
& \left.-11381760 \zeta^{2}(3)-7461168 \zeta(3)-1125439\right] / 403107840
\end{aligned}
$$

Knowledge of Eq. (3) means that $g_{c}$ is available at $O\left(1 / N^{3}\right)$ to all orders in $\epsilon$ and therefore we can proceed in deducing coefficients in the series of $\gamma(g)$, in 
a form similar to Eq. (4). The expression for $\eta=2 \gamma\left(g_{c}\right)$ at $O\left(1 / N^{3}\right)$, however, involves the $\epsilon$-expansion of a 2-loop self energy integral whose evaluation in terms of standard integration techniques for massless Feynman diagrams such as uniqueness is not possible for arbitrary $d$ Using group theory, for example, it has recently been written in terms of an ${ }_{3} F_{2}$ hypergeometric function 3 Therefore, writing the perturbative form of $\gamma(g)$ as

$$
\gamma(g)=\sum_{r=1}^{\infty}\left(c_{r} N^{2}+d_{r} N+e_{r}\right) N^{r-2} g^{r+1}
$$

with $e_{1} \equiv 0$ where we have only indicated the leading three orders in the $1 / N$ expansion, we deduce that the first appearance of a non-zeta number at this order in $1 / N$ is in $e_{8}$ or ninth loop. Explicitly

$$
\begin{aligned}
e_{8}=[ & 1044119552 \zeta(9)-2854457088 \zeta(8)+2246396928 \zeta(7) \\
& +264929280 \zeta(6) \zeta(3)+572052480 \zeta(6)+77856768 \zeta(5) \zeta(4) \\
& -663797760 \zeta(5) \zeta(3)-887356416 \zeta(5)-127844352 \zeta^{2}(4) \\
& +309288960 \zeta(4) \zeta(3)-476930304 \zeta(4)-8978432 \zeta^{3}(3) \\
& +286906368 \zeta^{2}(3)+677644800 \zeta(3) \\
& \left.+12386304 U_{62}-96663009\right] / 216710774784
\end{aligned}
$$

In Eq. (7) $U_{62}$ is the level 8 non-zeta number first discovered in Ref. [11]. In the $\beta$-function calculation it occurs at sixth loop and is associated with the $8_{19}=(4,3)$ torus knot

\section{QCD $\beta$-function}

We now report on a similar calculation for the $\beta$-function of QCD but at $O\left(1 / N_{f}\right)$ where $N_{f}$ is the number of quark flavours. An essential feature of the previous section was the equivalence of $\phi^{4}$ theory at the fixed point to another model. The property of universality meant that exponents calculated in either model gave the same results. This principle can be exploited in the case of QCD. In large $N_{f}$ it has been demonstrated, at_least in leading order, that QCD is equivalent to a lower dimensional model 12 Therefore it is possible to compute $d$-dimensional critical exponents in either model but the coefficients of the perturbative renormalization group functions of QCD are deduced from knowledge of the location of the QCD fixed point. The main advantage of using this equivalence in this instance is that the lower dimensional model has a simpler form. In particular it has fewer interactions which immediately 
reduces the number of Feynman diagrams which need to be computed. More concretely the lagrangian for QCD, in standard notation, is

$$
L=i \bar{\psi}^{i I} \not D \psi^{i I}-\frac{\left(G_{\mu \nu}^{a}\right)^{2}}{4 e^{2}}
$$

The model to which it is equivalent as $N_{f} \rightarrow \infty$ is the non-abelian Thirring model, (NATM), which is perturbatively renormalizable in strictly two dimensions and has the lagrangian,

$$
L=i \bar{\psi}^{i I} \not p \psi^{i I}-\frac{\left(A_{\mu}^{a}\right)^{2}}{2 \lambda}
$$

where the coupling constant $\lambda$ is dimensionless in 2-dimensions. Clearly the NATM contains only a single quark gluon interaction. In using Eq. (9) to compute exponents there would appear to be no contributions to quantities in QCD which are built with the triple and quartic gluon self-interactions. It was demonstrated, however 12 that these pieces are contained in subdiagrams which involve a quark loop which connects three and four gluon lines respectively. In other words using the NATM with the critical point massless propagators diagrams with such loops reproduce the contribution to the perturbation theory in QCD of both the loop itself and also the gluon self-interactions. Indeed this is apparent in the results we have previously computed using Eq. (9) in relation to the known explicit 3-loop $\overline{\mathrm{MS}}$ results] Further in dealing with nonabelian gauge theories we use a covariant gauge fixing. Therefore ghost fields must also be included in both lagrangians. It turns out that since a loop of ghost fields gives one power less of $N_{f}$ than at leading order in $1 / N_{f}$ they do not contribute to the exponents.

To find $\omega$ one computes the dimension of the operator associated with that coupling From Eq. (8) this is $G=\left(G_{\mu \nu}^{a}\right)^{2}$ and from the last term of Eq. (8) it is easy to deduce the scaling law which relates $\omega$ to the anomalous dimension of $G$ and the gluon anomalous dimension As the $O\left(1 / N_{f}\right)$ result for the abelian sector has been reported previously 1314 all that needs to be computed are the diagrams in the gluon 2-point function with $G$ inserted whose colour factor is proportional to $C_{2}(G)$. There are two such graphs; one two loop and one three loop. Calculating these with the massless critical point propagators we obtain the result

$$
\begin{aligned}
\omega_{1}=- & {\left[(2 \mu-3)(\mu-3) C_{2}(R)\right.} \\
& \left.-\frac{\left(4 \mu^{4}-18 \mu^{3}+44 \mu^{2}-45 \mu+14\right) C_{2}(G)}{4(2 \mu-1)(\mu-1)}\right] \frac{\eta_{1}^{\mathrm{O}}}{T(R)}
\end{aligned}
$$


This has been computed in an arbitrary covariant gauge and we have checked that the gauge parameter vanishes as it ought. Except in the Landau gauge, however, there is potential for the operator $G$ to mix with $\left(\partial^{\mu} A_{\mu}^{a}\right)^{2}$. It turns out that the mixing matrix at criticality is in fact diagonal. The final check we have is in relation to the $\epsilon$-expansion of Eq. (10) and we record that it agrees with the 3-loop $\overline{\mathrm{MS}}$ QCD $\beta$-function 2 . 2 . 17 This agreement further justifies our previous remarks concerning the use of the NATM in computing exponents in QCD. In similar notation to Eq. (4) we deduce the new higher order coefficients

$$
\begin{aligned}
a_{4}= & -\left[154 C_{2}(R)+53 C_{2}(G)\right] / 3888 \\
a_{5}= & {\left[(288 \zeta(3)+214) C_{2}(R)+(480 \zeta(3)-229) C_{2}(G)\right] / 31104 } \\
a_{6}= & {\left[(864 \zeta(4)-1056 \zeta(3)+502) C_{2}(R)\right.} \\
& \left.+(1440 \zeta(4)-1264 \zeta(3)-453) C_{2}(G)\right] / 233280 \\
a_{7}= & {\left[(3456 \zeta(5)-3168 \zeta(4)-2464 \zeta(3)+1206) C_{2}(R)\right.} \\
& \left.+(5760 \zeta(5)-3792 \zeta(4)-848 \zeta(3)-885) C_{2}(G)\right] / 1679616
\end{aligned}
$$

\section{Conclusion}

We have provided the two leading $\overline{\mathrm{MS}}$ numbers in the quintic in $N$ which appears as the coefficient in the $\beta$-function of $\phi^{4}$ theory at six loops. Although information on the hardest diagrams has been determined in the $N=1$ case而 computation of the remaining numbers to complete the six loop term is still far off. For this to be achieved a very high degree of ingenuity will be required to produce an algorithm which can then be implemented in some symbolic manipulation programme.

\section{Acknowledgements}

The work reported in this article has been carried out in part with the support of a PPARC Advanced Fellowship. The calculations were performed with the use of REDUCE version 3.4 and Form version 2.2c.

\section{References}

1. D.J. Broadhurst and D. Kreimer, Int. J. Mod. Phys C6, 519 (1995).

2. D. Kreimer, Phys. Lett. B354, 117 (1995).

3. D.J. Broadhurst, J.A. Gracey and D. Kreimer, hep-th/9607174.

4. A.N. Vasil'ev, Yu.M. Pis'mak and J.R. Honkonen, Theor. Math. Phys. 50, 127 (1982). 
5. J.A. Gracey, Phys. Lett. B373, 178 (1996).

6. A.N. Vasil'ev, Yu.M. Pis'mak and J.R. Honkonen, Theor. Math. Phys. 46, 157 (1981); 47, 465 (1981).

7. J.A. Gracey, in New Computing Techniques in Physics Research IV ed. B. Denby and D. Perret-Gallix (World Scientific, Singapore, 1995), p. 259.

8. J. Zinn-Justin, Quantum Field Theory and Critical Phenomena (Clarendon Press, Oxford, 1989).

9. J.A. Gracey, Int. J. Mod. Phys. A9, 567 (1994).

10. H.Kleinert, J. Neu, V. Schulte-Frohlinde, K.G. Chetyrkin and S.A. Larin, Phys. Lett. B256, 81 (1991); B319, 545 (1993).

11. D.J. Broadhurst, Z. Phys C41, 81 (1988).

12. A. Hasenfratz and P. Hasenfratz, Phys. Lett. B297, 166 (1992).

13. A. Palanques-Mestre and P. Pascual, Commun. Math. Phys. 95, 277 (1984).

14. J.A. Gracey, Int. J. Mod. Phys. A8, 2465 (1993).

15. D.J. Gross and F.J. Wilczek, Phys. Rev. Lett. 30, 1343 (1973); H.D. Politzer, Phys. Rev. Lett. 30, 1346 (1973).

16. W.E. Caswell, Phys. Rev. Lett. 33, 244 (1974); D.R.T. Jones, Nucl. Phys. B75, 531 (1974).

17. O.V. Tarasov, A.A. Vladimirov and A.Yu. Zharkov, Phys. Lett. B93, 419 (1980); S.A. Larin and J.A.M. Vermaseren, Phys. Lett. B303, 334 (1993). 